References

1 Ochroch AE, Kush S, Stuart S, Hollander JE, Levitan $R M$. Assessment of laryngeal view in direct laryngoscopy: the percentage of glottic opening (POGO) score compared to Cormack and Lehane grading. Can J Anesth 1999; 46: 987-90.

2 Rose DK, Cohen MM. The incidence of airway problems depends on the definition used. Can J Anaesth 1996; 43: 30-4.

3 Adnet F, Borron SW, Racine SX, et al. The intubation difficulty scale (IDS): proposal and evaluation of a new score characterizing the complexity of endotracheal intubation. Anesthesiology 1997; 87: 1290-7.

4 Levitan RM. Direct laryngoscopy imaging: teaching and research applications. Educational Synopses in Anesthesiology and Critical Care Medicine (June, 1998): The On-line Anesthesia Journal, http://gasnet.med.yale.edu/esia/1998/june/samart.html. Reprinted: American Journal of Anesthesiology 1999; 26: 39-42.

5 Benumof JL. Intubation difficulty scale: anticipated best use (Editorial). Anesthesiology 1997; 87: 1273-4.

\section{Transesophageal echocardiography in the management of anaphylactic shock}

To the Editor:

We would like to present the use of transesophageal echocardiography (TEE) in the management of anaphylactic shock.

After infusion of dextran, a 47-yr-old man suffered an anaphylactic reaction, consisting of hypotension and bronchospasm. Prednisolone and aminophylline were given and, after midazolam and rocuronium, the trachea was intubated. Large amounts of pink frothy fluid appeared in the endotracheal tube. In the ICU, mechanical ventilation was started with PEEP of $16 \mathrm{~cm}$ $\mathrm{H}_{2} \mathrm{O}$. A pulmonary artery catheter was inserted and a CVP of $20 \mathrm{mmHg}$ and pulmonary capillary wedge pressure (PCWP) of $26 \mathrm{mmHg}$ were measured. Chest $\mathrm{X}$-ray showed massive pulmonary edema. Continuous infusion of epinephrine was started to maintain blood pressure (BP), but without success.

A TEE examination showed good myocardial contractility and no cardiac tamponade. Ventricular dimensions were small. However, CVP and PCWP were elevated, indicating a small circulating blood volume in a contracted vascular system, caused by massive extravascular fluid trapping in the lungs and vasopressor-induced vasoconstriction. Under TEE monitoring,
$1500 \mathrm{ml}$ of a gelatin colloid were infused until the ventricular dimensions appeared normal. The $\mathrm{BP}$ and $\mathrm{SaO}_{2}$ improved to normal, while PAP and PCWP remained unchanged. Epinephrine was discontinued and hemodynamic variables remained stable.

Although infusion of fluids is generally necessary to treat hypotension during anaphylactic shock, we felt this may be hazardous in the presence of high PCWP and pulmonary edema. TEE was helpful in evaluating the intravascular volume status, and complemented pressure measurements of the PA catheter.

W. Anton Visser

Frederik W. Santman

Helmut R. Gehlmann

Wim R.M. Aengevaeren

Nijmegen, The Netherlands

\section{Blind intubation using the Endotrol tube and a light wand}

To the Editor:

The Endotrol tracheal tube (Mallinckrodt, Athlone, Ireland) is useful for blind nasotracheal intubation, since the curve of the tube can be controlled by a wire hook. ${ }^{1,2} \mathrm{We}$ suggest that the use of a light wand (Trachlight [Laerdal Medical, New York, USA]) adds to the usefulness of the Endotrol tube.

A 25-yr-old woman had a fractured jaw a few months ago and was scheduled for reconstruction of the jaw. Preoperatively, the mouth opening was restricted to two-fingers width. General anesthesia was induced, and after adequate ventilation was confirmed vecuronium was injected. An Endotrol tube was inserted into the nose and oral laryngoscopy was attempted. Since the glottis could not be seen, blind tracheal intubation was attempted. However, location of the glottis was difficult due to the lack of spontaneous breathing, and intubation failed after three attempts.

Trachlight-aided blind nasotracheal intubation was then attempted while a fibreoptic bronchoscope was being prepared. After the inner metal stylet has been removed from the Trachlight, the wand was inserted into the Endotrol tube. By advancing the tube, illumination was seen in the side of the neck. This time, the tube position could easily be adjusted to the centre by rotating the tube and pulling the hook. The tube was further advanced, the wand was removed and the correct position was confirmed. The time for tracheal intubation was less than $30 \mathrm{sec}$. 
Takashi Asai

Koh Shingu

Osaka, Japan.

\section{References}

1 Hooker EA, Hagan S, Coleman R, Heine MF, Greenwood P. Directional-tip endotracheal tubes for blind nasotracheal intubation. Acad Emerg Med 1996; 3: 586-9.

2 Asai T. Endotrol tube for blind nasotracheal intubation (Letter). Anaesthesia 1996; 51: 507. 